Rev. Biol. Trop., 47(4): 1035-1038, 1999

www.ucr.ac.cr www.ots.ac.cr www.ots.duke.edu

\title{
Búsqueda de alimento y marcación de trillo por larvas de Brassolis sophorae (Lepidoptera: Nymphalidae) en condiciones naturales
}

\author{
Ronald Zanetti ${ }^{1}$, Evado F. Vilela ${ }^{2}$, José C. Zanuncio ${ }^{2}$ y José Inácio L. Moura ${ }^{3}$ \\ 1 Universidade Federal de Lavras, Departamento de Entomologia. Caixa Postal 37, 37200-000, Lavras, Minas Gerais, \\ Brasil. Fax: 5535 8291288, correo electrónico: zanetti@ufla.br. \\ 2 Universidade Federal de Viçosa, Departamento de Biologia Animal. 36571-000 Viçosa, Minas Gerais, Brasil. \\ 3 CEPEC/CEPLAC. 45600-000 Caixa Postal 07 Itabuna, Bahia, Brasil.
}

Recibido 3-XII-1998. Corregido 12-V-1999. Aceptado 20-V-1999.

\begin{abstract}
The daily periodicity of foraging and trail marking behaviour of Brassolis sophorae larvae was studied between August, 1991 and July, 1992 in a cocoa plantation in Bahia, Brazil. Larvae, originating from eight different nests, were observed continually during the dark phase for 20 consecutive days. They foraged for a single period of time each day, starting at 17:56 h, and for a meam duration of $90 \mathrm{~min}$ and $36 \mathrm{~s}$. Larvae mark trails with threads of silk while moving from the nest to the foraging site and viceversa.
\end{abstract}

Key words: Foraging behaviour, trail marking, Brassolis sophorae, Lepidoptera, Nymphalidae.

Las especies de insectos pre-sociales utilizan la comunicación por medio de sustancias químicas para mantenerse organizadas (Fitzgerald 1976). Los lepidópteros son citados como ejemplos de estos insectos, destacándose, principalmente, especies del género Malacossoma (Lepidoptera: Lasiocampidae) (Fitzgerald 1982), conocidas por utilizar trillos impregnadas con feromona para orientar a los individuos durante la búsqueda de alimento. Se recluta a los individuos hambrientos, hacia lugares adecuados de alimentación, en un comportamiento similar al de algunas hormigas.

El defoliador de las palmeras, Brassolis sophorae (L., 1758) (Lepidoptera: Nymphalidae), también presenta comportamiento presocial. Las larvas son gregarias y construyen un nido, uniendo diversos foliolos de palmeras por medio de hilos de seda, allí pasan el día en reposo y salen solamente en la noche para alimentarse (Mariconi 1952).

En el presente estudio se investigan el período diario de alimentación y el comportamiento de marcación de trillo de las larvas de B. sophorae, en condiciones de campo.

Los estudios fueron conducidos en una plantación de cocotero variedad enano verde de cuatro años de edad, en Una-Bahia, entre agosto de 1991 y julio de 1992 . Se recolectó ocho nidos de $B$. sophorae con larvas de $4^{\circ}$ estadio. Las larvas se transfirieron a un nido artificial, constituído por dos potes plásticos de $500 \mathrm{ml}$, unidos por la boca y envueltos por un plástico oscuro y opaco. Cada nido poseía un 
orificio de $1.5 \mathrm{~cm}$ de diámetro en cada extremidad, de modo que el orificio superior dada paso a las larvas y por el inferior salían los excrementos. Ocho nidos artificiales fueron fijados basalmente en foliolos de cocoteros (tres cocoteros contenían dos nidos cada uno, instalados en lados opuestos y los otros dos cocoteros contenían solamente un nido).

Se observó las larvas continuamente durante la escotofase, por 20 días consecutivos, a partir de la colocación de los nidos en los cocoteros. El inicio y la finalización del forrajeo eran marcados por la salida y entrada de la primera larva en el nido, independientemente de cual individuo fuera.

Los datos excluyen el dia inicial (establecimiento larval) y los cuatro dias anteriores a la muerte de larvas muertas por hongos, pues según Habib y Andrade (1977) las larvas de $B$. sophorae contaminadas presentan movi- mientos lentos y no se alimentan 24 horas después de la contaminación, muriendo al cuarto dia.

Para determinar si las larvas siguen trillos hechos por otras, el orificio de salida del nido se cerró después de la salida de una larva. Se verificó su ruta en una hoja no explorada anteriormente, hasta el inicio de la alimentación, y luego se liberó individualmente otras larvas del nido $(\mathrm{N}=4)$ para registrar si seguín la misma ruta.

No hubo diferencia significativa entre los promedios de los ocho nidos en cuanto a los parámetros observados (Cuadro 1). Las larvas presentaron un único período diario de búsqueda de alimento, que se inicia a las $17 \mathrm{hr}$ $56 \mathrm{~min}$ ( $17 \mathrm{hr} 50 \mathrm{~min}$ guion $18 \mathrm{hr} 05 \mathrm{~min}$ ) y dura $90 \mathrm{~min}$ y $36 \mathrm{~s}$ (74min-105min). Después de la alimentación las larvas permanecen inactivas por, aproximadamente, $22 \mathrm{hr} 30 \mathrm{~min}$, hasta el inicio del próximo periodo.

\section{CUADRO 1}

Horarios medios de inicio, término y duración del período de forrajeo de larvas de Brassolis sophorae. Una, Bahia. Cada fila es un nido*.

$\begin{array}{ccc} & \text { Días considerados } & \text { Hora de inicio } \\ & 13 & 17: 50 \\ 16 & 17: 59 \\ 15 & 18: 05 \\ 11 & 17: 53 \\ 10 & 18: 04 \\ 14 & 17: 54 \\ & 09 & 17: 56 \\ & 11 & 17: 52 \\ \text { Media } & 12.38 & 17: 56\end{array}$

$\begin{array}{cc}\text { Hora de finalización } & \text { Duración (min) } \\ 19: 32 & 102 \\ 19: 26 & 87 \\ 19: 32 & 87 \\ 19: 15 & 82 \\ 19: 35 & 91 \\ 19: 08 & 74 \\ 19: 41 & 105 \\ 19: 29 & 97 \\ 19: 27 & 90\end{array}$

* Medias de las columnas no diferen entre si $(\mathrm{F} \mathrm{p}>.05)$.

Brassolis isthmia Bates (Lepidoptera: Nymphalidae) también presenta un único período de alimentación diario, que se inicia entre las $17 \mathrm{hr} 00 \mathrm{~min}$ y $17 \mathrm{hr} 30 \mathrm{~min}$ y dura, aproximadamente, $45 \mathrm{~min}$. Asi omo B. isthmia, B. sophorae también se alimenta de noche lo que es bastante característico en los brasolídeos (Young 1985).
Los nidos artificiales no fueron adecuados, pues siete de los ochos nidos instalados en los cocoteros fueron abandonados. Las larvas siguieron hasta el nuevo local de nidificación, generalmente, en la base de las hojas de las respectivas plantas. Una vez establecidas, las larvas salieron y permanecieron próximas a la entrada, depositando hilos de seda. Después de 
algunos minutos, comenzaron a dejarlo en uno o más grupos, generalmente en sentido ascendente (abajo o encima de la nervadura principal de la hoja). Una larva seguía al frente de cada grupo y depositaba un hilo de seda en zig-zag oscilando la cabeza (ver Zamith 1962). Este comportamiento fue imitado por las larvas que la seguían formando, asi, un trillo de hilos, en forma de red, del nido hasta el lugar de alimentación.

$\mathrm{Al}$ avanzar sobre una hoja inexplorada, las larvas deambulan y frecuentemente la primera se devuelve y es substituída. El grupo avanza aunque se retire el líder ( $\mathrm{N}=3$ ), como en Malacossoma americanum Fabr. (Lepidoptera: Lasiocampidae) (Fitzgerald 1983) y en contraste com Malacossoma disstria Hübner (Lepidoptera: Lasiocampidae) (Greenblat y Witter 1976).

La búsqueda de alimento fue de la punta hacia la base del foliolo y de la hoja. Cuando la primera larva llegó a la punta del foliolo comenzó a alimentarse, las demás se detuvieron inmediatamente atrás de ella, tocándose lateralmente y se alimentaron com los lados del foliolo que, generalmente, se dobla por al peso de las larvas. Cuando un foliolo estaba completamente ocupado la mayoría se dirigio a otro (generalmente adyacente).

Las larvas depositaron seda en la superficie interna del nido inmediatamente antes y después de cada salida. Sellan la entrada con seda, lo que probablemente evita la entrada de parasitoides o depredadores (en dos nidos el tapón tenia un pequeño orificio, aproximadamente igual al diámetro de una larva, el cual es obstruído por la cabeza de una de ellas). Las larvas aparentemente no distinguen a sus compañeras de nido pues si se coloca experimentalmente juntas larvas de dos nidos se alimentan normalmente y se alojan en un mismo nido.

Para el regreso se deposita una nueva red de seda sobre el trillo. La red nueva, más húmeda, atrae larvas que aún no se habían alimentado al igual que en otros lepidópteros pre-sociales, como M. americanum (Fitzgerad 1976, 1980) y M. disstria (Greenblat y Witter 1976, Fitzgerald y Costa 1986).
Algunas larvas solitarias se detenían al retornar, hasta ser tocadas por otra larva; entonces proseguían juntas hasta el nido. Este comportamiento fue menos común cuando la larva exploraba un nuevo lugar. Basta una larva para marcar trillos de exploración y reclutamiento, que fueron seguidos, hasta el extremo por $88.2 \%$ de sus compañeras (Cuadro 2), sin necesidad de contacto físico entre ellas. Las larvas de $B$. sophorae marcan y siguen trillos, igual que otras espécies de lepidópteros pre-sociales.

\section{CUADRO 2}

Porcentaje de larvas de Brassolis sophorae que siguieron trillos depositadas por otras. Una, Bahia.

$\begin{array}{ccc} & \text { Larvas por Nido } & \text { Número } \\ & 80 & 74 \\ & 67 & 58 \\ & 62 & 49 \\ & 71 & 66 \\ \text { Total } & 280 & 247\end{array}$

\section{AGRADECIMIENTOS}

Los autores agradecem al Conselho Nacional de Desenvolvimento Científico e Tecnológico (CNPq) e la Comissão Executiva do Plano da Lavoura Cacaueira (CEPLAC), por la ayuda concedida para la ejecución de este trabajo.

\section{REFERENCIAS}

Fitzgerald, T.D. 1976. Trail marking by larvae of the eastern tent caterpillar. Science 194: 961-963.

Fitzgerald, T.D. 1980. An analysis of daily foraging patterns of laboratory colonies of the eastern tent caterpillar, Malacossoma americanum (Lepidoptera: Lasiocampidae), recorded photo-electronically. Can. Entomol. 112: 731-738.

Fitzgerald, T.D. 1982. Exploratory and recruitment trail marking by eastern tent caterpillars, p. 111-119. In J.R. Matthews \& R.W. Matthews (eds.). Insect behavior: a sourcebook of laboratory and field exercises. Westview, Colorado. 
Fitzgerald, T.D. 1983. Caterpillar on a silk thread. J. Natur. Hist. 2: 57-62.

Fitzgerald, T.D. \& J.T. Costa. 1986. Trail-based communication an foraging behavior of young colonies of forest tent caterpillars (Lepidoptera: Lasiocampidae). Ann. Entomol. Soc. Amer. 76: 999-1007.

Greenblat, J.A. \& J.A. Witter. 1976. Behavioral studies on Malacossoma disstria (Lepidoptera: Lasiocampidae). Can. Entomol. 108: 1225-1228.

Habib, M.E.M. \& C.F. Andrade. 1977. Epizootia em larvas de Brassolis sophorae (Linnaeus, 1758) causada por Beauveria bassiana, (Bals.) Vuil., com estudos de identificação e sintomatologia. An. Soc. Entomol. Bra. 6; 239-247.
Mariconi, F.A.M. 1952. As lagartas das palmeiras. ABrassolis sophorae (Lineu, 1758); B-Brassolis astyra Godart, 1821. O Biológico 18: 103-107.

Young, A.M. 1985. Natural history notes on Brassolis isthmia Bates (Lepidoptera: Nymphalidae: Brassolinae) in northeastern Costa Rica. J. Res. Lep. 24: 385-392.

Zamith, A.P.L. 1962. Anatomia morfológica, externa e interna da lagarta, e do adulto de Brassolis sophorae laudrentii Stichel, 1925. An. Escol. Sup. Agr. "Luiz de Queirós" 19: 431-451. 